\title{
Zur Frage \\ über die Wirkung rascher Veränderungen des Luftdruckes auf den Organismus.
}

Von

E. von Cyon.

In diesem Archiv (Bd. 67 1. u. 2. Heft) haben Dr. Richard Heller, Dr. Wilhelm Mayer und Dr. Hermann on Schrötter eine grosse Reihe von Untersuchungen mitgetheilt, welche die im Titel bezeichnete Frage behandeln. Die Wirkungen der Luftdruckveränderungen auf den Organismus haben bekanntlich ausser einem rein therapeutischen Interesse noch eine grosse praktische Wichtigkeit für Leute, die bei Tunnelarbeiten und in Minen beschäftigt sind, oder Bergbesteigungen und Luftschifffahrten unternehmen. Fùr diese Leute ist eine streng wissenschaftliche Prüfung dieser Wirkungen, im wahren Sinne des Wortes, eine Lebensfrage. Es genügt nur an das tragische Ende der beiden Luftschifffahrer CroceSpinelli und Syvel zu erinnern, welche ihr Veitrauen zu den leichtsinnigen und unwissenschaftlichen Betheuerungen Paul Bert's mit dem Leben büssten.

Nur in Anbetracht dieser ausserordentlichen praktischen Wichtigkeit der hier in Betracht kommenden Fragen erlaube ich mir, zu der Arbeit von Dr. Heller, Dr. Mayer und Dr. von Schrötter einige Bemerkungen zu machen. Diesen sind wahrscheinlich meine Untersuchungen über die Wirkung hoher barometrischer Drucke auf die Organe der Respiration und Circulation ${ }^{1}$ ) ganz entgangen. Diese Untersuchungen, ausgeführt im Laboratorium von Paul Bert, zum Theil mit Hülfe der von ihm für seine Versuche construirten Apparate, haben bekanntlich auf das Evidenteste erwiesen, dass seine Apparate für die Fragen, welche Paul Bert entscheiden

1) Comptes rendus de l'Académie des Sciences de Paris 1882; Archiv für Physiologie von Du Bois-Reymond 1884 (Jubelband) und meine „Gesammelte Physiologische Arbeiten", Berlin, Hirschwald'sche Buchhandlung 1888. 
Zur Frage üb. d. Wirkung rascher Veränderungen d. Luftdr., a. d. Organismus. 93

wollte, meistens ganz unbrauchbar waren, dass seine Versuche voll der gröbsten Fehler waren, seine Gasanalysen nach ganz unwissenschaftlichen Methoden ausgeführt wurden, und endlich, dass seine ganz willkürlichen Schlüsse im grellen Widerspruche mit seinen eigenen Versuchsergebnissen standen. Meine Einwände und experimentellen Belege für die Untauglichkeit der Pau1 Bert'schen, „von schülerhaften Fehlern behaftetèn Arbeit", wie sich Du BoisReymond schonend ausgedrückt hat, waren der Art, dass man hoffen könnte, dass Forscher, welche von Neuem an das Studium der wichtigen Frage über die Wirkungen hoher atmosphäriseher Drucke gehen, es sorgfältig vermeiden werden, die Paul Bertschen Arbeiten zum ernsten Ausgangspunkt für neue Versuche zu machen. Bekanntlich hat Paul Bert darauf verzichtet, die unmögliche Vertheidigung seines "berühmten" Werkes "La pression barométrique" zu übernehmen.

Er vermied es einfach, nach dem Erscheinen meiner Kritik in seinem Laboratorium zu erscheinen, und hat nie mehr etwas Wissensehaftliches zu veröffentlichen gewagt. Er zog es vor, nach Tonkin zu gehen, wo er noch bei den Annamiten als gelehrter occidentaler "Mandarin" figuriren konnte. Bald darauf starb er.

In Deutschland scheinen aber die P. Bert'sehen Arbeiten noch eine gewisse Autorität zu besitzen. Es ist daher leicht verständlich, dass Dr. Heller, Dr. Mayer und Dr. von Schrötter, indem sie von Neuem Untersuchungen über eine so wichtige Frage anstellten, sich Paul Bert zum Muster genommen haben, zuerst in dem Bau der Apparate und sodann leider auch in der Art, gasometrische Messungen auszuführen. Zu letzteren benutzten sie zwar die vortrefflichen Methoden von Pflüger, welche ich in meiner Arbeit $\mathrm{Paul} \mathrm{Bert}$ als Muster vorschlug; sie haben aber die Genauigkeit ihrer Messungen durch die Wahl der zur Gasbestimmung benutzten Blutmengen bedeutend verringert. So z. B. schreiben sie (1. c. S. 43): „Des Ferneren wird man es nicht als einen groben Fehler bezeichnen können, dass wir, um eine möglichst grosse Gasmenge zur Analyse zu bekommen, das Gas von mehreren Versuchsthieren in einem Rohr gesammelt und untersucht haben; werden doch jedenfalls die gefundenen Zahlen brauchbare Mittelwertbe darstellen, welche uns über die Zusammensetzung der nach raschem Druckabfall im Gefäss-Systeme auftretenden freien Gasblasen Aufschluss geben." 


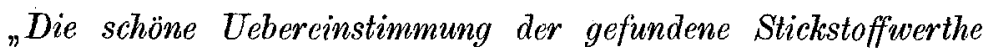
mit den von uns und von P. Bert bestimmten Zahlen spricht für die gewonnenen Resultate."

Wenn man weiss, welche primitiven Methoden P. Bert, „ein Feind der Decimalstellen und ein Verächter der Logarithmentafeln" ${ }^{1}$ ), zu seinen Gasanalysen gebraucht hat, so kann diese "schöne Uebereinstimmung" nur als ein ominöses Zeichen gelten. Liest man die zahlreichen Versuche, die unter meistens abweichenden Umständen von diesen Autoren angestellt worden sind, so muss man, entgegen ihrer eigenen Ueberzeugung, die Sammlung „des Gases von mehreren Versuchsthieren" doch als einen Fehler betrachten.

Das Vertrauen dieser Autoren in die Unfehlbarkeit Paul Bert's ist um so auffallender, als sie ja selbst angeben, van Rensselaer (1891) und Clark (1893) hätten gezeigt, dass „die Sätze Paul Bert's und seiner Schule einer eingehenderen Kritik nicht Stand halten".

Dr. Heller, Dr. Mayer und Dr. von. Schrötter haben zweierlei Compressionsapparate zu ihren Versuchen benutzt, von denen sie nur einen schon jetzt beschrieben haben. Dieser Apparat, die Experimentalkammer, ist, nach der Beschreibung und der gelieferten Zeichnung zu urtheilen, ganz nach dem Modell gebaut worden, welchen P. Bert auf S. 654 seines Buches beschrieben hat, und dessen auch ich mich bei der Mehrzahl meiner Versuche bedient habe. Nur ist der Apparat dieser Herren um 1/2 m länger, $2 \mathrm{~m}$ anstatt $1^{1 / 2}$ bei $\mathrm{P}$. Bert. Ich habe in meiner Arbeit die Uebelstände dieses Apparates ausführlich beschrieben und brauche nicht nochmals darauf zurückzukommen. Die Schwierigkeiten, am Thier zu experimentiren, während es in diesem Cylinder eingeschlossen ist, scheinen bei dem ihrigen noch beträchtlicher zu sein. Ich vermochte mit dem Bert'schen Apparat doch wenigstens ein Manometer mit der Carotis des Thieres in Verbindung zu bringen und die Athmungs- und Circulationsveränderungen während der Versuche genau zu messen, ja, dabei sogar Reizungen von Nerven (ischiadicus und tibialis) an den Thieren vorzunehmen, während Dr. Heller, Dr. Mayer und Dr. von Schrötter erklären (S. 72) dass sie bei ihren Versuchen weder ein Ludwig'sches Manometer mit Kymographion, noch überhaupt eine Ludwig'sche Röhre zur Messung des

1) Siehe meine "Gesammelte Arbeiten" etc. S. 158 u. f. 
Blutdrucks verwenden konnten. Sie mussten sich mit der Benutzung des v. Basch'schen Sphygmomanometers begnügen.

Noch viel unvortheilhafter stellen sich die Apparate dieser Herren heraus, im Vergleich zu der grossen Bert'schen Kammer, in welcher ich mich bequem mit allen denjenigen Instrumenten und Apparaten einschliessen konnte, welche für hämodynamische Versuche erforderlich waren (1. c. S. 169 und folgende). Ich konnte also nicht nur an Versuchsthieren, sondern an mich selbst Beobachtungen über die Wirkungen hoher Drucke anstellen.

Ihre Compressionsapparate waren also für präcise physiologische Versuche ebenso wenig brauchbar wie die Bert'schen. Wir wollen nun ihre Versuchsweise selbst an einigen Beispielen prüfen. Der Zweck ihrer Versuche bestand in der Bestimmung des Einflusses, welchen hohe Drucke auf die Blutvertheilung im Gehirne und in den anderen inneren Organen auszuüben vermögen. Ein Kaninchen wird in die "Sanitätsschleuse" gebracht. Ich citire wörtlich:

„Versuch I. Zeit der Compression: 27 Minuten. Druck 2,1 Atmosphäre. Dauer des Aufenthaltes: $3 / 4$ Stunde. Zeit der Decompression: 50 Minuten. Eine balbe Stunde nach erreichtem Maximaldrucke, wird das Kaninchen durch Schläge auf den Kopf getödtet und secirt."

Abgesehen davon, dass 27 Minuten plus 50 Minuten nicht $3 / 4$ Stunde ausmachen, ist die Tödtungsweise der Thiere in der "Sanitätsschleuse" durch Schläge auf den Kopf doch ein kaum zulässiges Verfahren, um festzustellen, ob die hohen Drucke nicht Congestionen im Gehirn veranlassen. Um so auffallender erscheint die Angabe, dass die Section "keine Congestion der inneren Organe oder des Gehirnes und Rückenmarkes ergeben“ hat! "Sowohl während des weitern Aufenthaltes in comprimirter Luft als auch beim Aussehleusen und nach der Decompression zeigten sich keine Veränderungen am getödteten Thiere" (S. 12).

Im Versuch II wird das Kaninchen ebenfalls durẹ Schläge auf den Kopf getödtet, und war „die Blutfüllung der Meningen gleich der des Thieres beim Versuch I.".

Diese Art der Tödtung ist die bei allen Versuchen an Kaninchen von den Autoren bevorzugte; nur ein paar Mal (Versuch 12 und 14) "wird das Thier durch Schläge ins Genick im Caisson getödtet." Bei ihren Versuchen mit Meerschweinchen verfuhren die Autoren in anderer Weise: Sie tödteten die Thiere erst $8 \mathrm{~T}$ ag e nach der Einwirkung der hohen Drucke, während 
welcher sich die "Thiere vollkommen wohl" befanden, und fanden bei der Section "normale Verhältnisse".

Nur in dem Gebörorgan der Meerschweinchen wären einige Veränderungen gefunden.

Für Hunde wurde der Tod durch Chloroformmarkose gewählt. Dies war vielleicht auch nicht die beste Weise, um die Blutvertheilung in den inneren Organen unter dem Einfluss hoher atmosphärischer Drucke zu beobachten, da Chloroform bekanntlich schon selbst nicht $\mathrm{zu}$ vernachlässigende Veränderungen in der Blutvertheilung zu erzeugen pflegt.

Aus 120 äbnlichen Versuchen ziehen die drei Autoren nun folgende Schlüsse:

"Die Blutvertheilung des Organismus erfäbrt in comprimirter Luft aus mechanischen Ursachen keine Veränderung." Dies ist sicherlich ein Irrthum. Schon Pa num hat auf die Störungen in der Blutvertheilung des Organismus aufmerksam gemacht, welche jn Folge der Unterschiede zwisehen der Elasticität der Lungen und derjenigen der Bauchwandungen entstehen müssen. Ich habe schon die mit blossem Auge sichtbaren Veränderungen in der Circulation der peripheren, Organe beschrieben und gleichzeitig die Erklärung gegeben, warum diese Veränderungen sich nicht immer durch manometrische Bestimmungen des Blutdruckes in der Carotis manifestiren (l. c. S. 171).

„Nach rascher Decompression, deren Dauer für den einzelnen Fall innerhalb einer gewissen unteren Grenze schwankt, kann man im Gefässsystem freies Gas nachweisen." Diese Thatsache ist ganz richtig. Schon im Jahre 1857 hat Hop pe-Seyler diese Thatsache festgestellt und auch als die einzige Todesursache bei raschem Absinken des barometrischen Druckes angegeben. Die Autoren kennen die Hoppe-Seyler'sche Arbeit und citiren sie ganz richtig. Sie ziehen es aber in Allem vor, mit $\mathrm{Pan} 1 \mathrm{Bert}$ in Uebereinstimmung zu sein. Sie schreiben daher, dass Paul Bert "unabhängig von Hoppe-Seyler im Jahre 1871" dasselbe gefunden hat. Woher wissen die Herren, dass Paul Bert unabbängig von HoppeSeyler zu diesem Schluss gelangt ist? Paul Bert selbst hat diẹs nie zu behaupten gewagt. "Schon Mermmod," schrieb ich in meiner Abhandlung ${ }^{1}$ ), "hat Paul Bert auf die Thatsache aufmerk-

1) I. c. S. 167 . 
sam gemacht, dass 16 Jahre vor ihm Hoppe-Seyler die Ursache des durch plötzliche Entlastung eintretenden Todes und die Hauptursache der Bergkrankheit entdeckt hatte, aber Paul Bert zieht sich aus der Schlinge, indem er erklärt, dass diese Prioritätsfragen immer nur ein mittelmässiges Interesse darbieten"" (S. 1158).

"War der Aufenthalt in comprimirter Luft ein genügender und die Decompression eine entsprechend rasche, so treten nach derselben pathologische Erscheinungen auf." Auch dies ist richtig, und habe ich schon bei meiner Kritik der Bert'schen Versuche aus seinen eigenen Versuchen bewiesen, dass es die plötzliche Entlastung bei seinen Versuchen war, welche die pathologischen Zufälle und sogar den Tod herbeiführte (S. 167). Die Beobachtungen von Heller, Mayer und v. Schrötter über die Natur dieser Zufälle am Herzen, an den Gefässen und den Athmungsorganen stehen dagegen im grellsten Widerspruch mit den Thatsachen. Und eben in dieser Beziehung liegt die grosse Gefahr ihrer Betrachtungen. Diese Forscher geben selbst zu, dass sie die nöthigen präcisen Messapparate bei ihren Beobachtungen nicht verwenden konnten, wegen Mangels an Raum in ihren Compressionskammern. Dies genügt vollständig, um ihre negativen Beobachtungen zu erklären; nur wäre eine grössere Zurückhaltung bei den Schlüssen geboten, in Anbetracht der ganz positiven Resultate, welche Andere und ich bei solchen Versuchen, dank dem Gebrauch präciser Beobachtungsmethoden erhalten haben.

Es genügt, einen Blick auf meine Tabellen zu werfen (S. 178 und f. Gesammelte Arbeiten), um sich davon zu überzeugen, mit welcher Präcision sich, sowohl die Zahl der Athembewegungen und der Herzschläge, wie die Höhe des Blutdrucks verändern mit der Zunahme des athmosphärischen Druckes. Sogar die momentane Rückkehr zur Norm, welche constant auftritt, sobald dieser Druck zwei Athmosphären erreicht beweist, wie gesetzmässig solche Veränderungen aufzutreten pflegen.

Ich habe bekanntlich die beobachteten Veränderungen auf die Abnahme der Kohlensäurespannung im Blute zurückgeführt. Nur bei einigen Erscheinungen, wie z. B. bei der Beschleunigung des Herzschlags, spielt wabrscheinlich die Zunahme der Sauerstoffspannung eine Rolle. In neuester Zeit hat Mos s o bei seinen Versuchen über die Bergkrankheit auf dem Monte-Rosa dieselben Veränderungen ebenfalls auf die Abnahme der Kohlensäure im Blute, dieses 
98 E. v. Cy on: Zur Frage üb. d. Wirk. rasch. Verändergn. d. Luftur. a. d. Organism. normalen Erregers der Athmungs-, Herz- und Gefässcentren, zurückgeführt ${ }^{1}$ ), also ganz in Uebereinstimmung mit uns und im Gegensatz zu Paul Bert.

Es ist die höchste Zeit, den Glauben an die physiologischen Phantasien Bert's, welche schon so vielen Menschen das Leben gekostet haben, gründlich zu zerstören. Seine ganz unerwarteten Verehrer haben ihre Untersuchungen noch nieht beendet und versprechen eine neue, ausführlichere Arbeit über diesen Gegenstand. Hoffentlich finden sie noch Zeit, um die hier angefübrten Bemerkungen zu berücksichtigen und, um in ihrer sonst sehr mühsamen und fleissigen Untersuchung noch die nothwendigen Verbesserungen zu machen.

1) Comptes rendus de la Soc. d. Biologie 27 Fèv. 1897. 